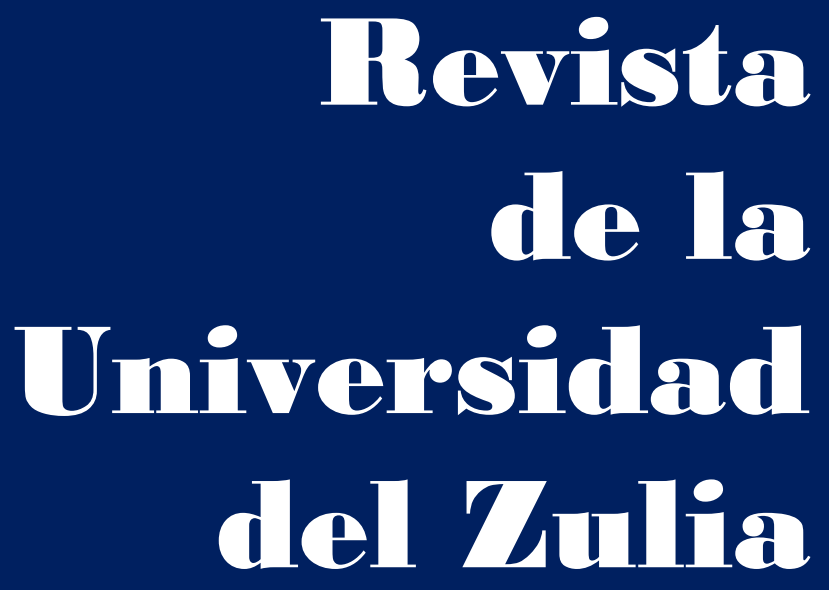

Fundada en 1947

por el Dr. Jesús Enrique Lossada

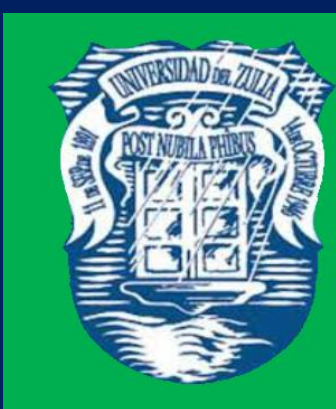

Ciencias del

Agred

Ingemieria

y Teenología
Añต 12 No 32

Enero - Abril 2021

Tercera Época

Maracailbo-Venezuela 
REVISTA DE LA UNIVERSIDAD DEL ZULIA. 3época. Año $12 \mathrm{~N}^{\circ}$ 32, 2021

Gelver Silva Valqui et al. // Influencia de sustratos en el crecimiento y desarrollo de tomate ...317-329

DOI: http://dx.doi.org/10.46925//rdluz.32.19

\title{
Influencia de sustratos en el crecimiento y desarrollo de tomate (Lycopersicum esculentum Mill) cultivado bajo un sistema hidropónico en invernadero
}

\author{
Gelver Silva Valqui* \\ Tito Sanchez Santillan** \\ Segundo Grimaldo Chavez Quintana**** \\ Jardy Chichipe Oyarce $* * * *$ \\ Segundo Manuel Oliva Cruz ${ }^{* * * *}$
}

RESUMEN

El presente trabajo tuvo por objetivo evaluar la influencia de sustratos en el crecimiento y desarrollo de tomate (Lycopersicum esculentum Mill) cultivado bajo un sistema hidropónico en invernadero. Se instaló bajo un diseño completo al azar (DCA), con cuatro tratamientos: $\mathrm{Tl}$ (75\% cascarilla de arroz $+25 \%$ arena, T2 (75\% cascarilla de arroz carbonizada $+25 \%$ arena, T3 (75 \% Aserrín $+25 \%$ arena, T4 (40\% cascarilla de arroz carbonizada $+35 \%$ aserrín $+25 \%$ arena); 8 repeticiones y 32 plantas. Se encontró que el T2, favoreció el incremento de altura $(139.13 \mathrm{~cm})$, diámetro de tallo $(10.95 \mathrm{~mm})$, número de tomates de primera calidad (15.25) y rendimiento (3.03 kg/planta); no obstante, el T3 y Tl mostraron baja influencia en dichas variables. Se concluye que, el tomate obtiene mayores características morfológicas y fisiológicas en sustrato (cascarilla carbonizada + arena); el aserrín como sustrato no es ideal para su crecimiento y desarrollo.

PALABRA CLAVE: cascarilla carbonizada; hidroponía; rendimiento de tomate; sustrato inerte; tecnología limpia

*Investigador del Instituto de Investigación para el Desarrollo sustentable de Ceja de Selva INDES-CES, Universidad Nacional Toribio Rodríguez de Mendoza de Amazonas, Perú. ORCID: https://orcid.org/0000-00021471-1810. E-mail: gelversilvavalqui28@gmail.com

***nvestigador del Instituto de Investigación para el Desarrollo sustentable de Ceja de Selva INDES-CES, Universidad Nacional Toribio Rodríguez de Mendoza de Amazonas, Perú. ORCID: https://orcid.org/0000-00020946-3445. E-mail: titosanchezsantillan@gmail.com

${ }^{* * *}$ Investigador del Instituto de Investigación para el Desarrollo sustentable de Ceja de Selva INDES-CES, Universidad Nacional Toribio Rodríguez de Mendoza de Amazonas, Perú. ORCID: https://orcid.org/0000-00020946-3445. E-mail: segundo.quintana@untrm.edu.pe

${ }^{* * * *}$ Investigador del Instituto de Investigación para el Desarrollo sustentable de Ceja de Selva INDES-CES, Universidad Nacional Toribio Rodríguez de Mendoza de Amazonas, Perú. ORCID: https://orcid.org/0000-00025913-2388. E-mail: jardy06@gmail.com

${ }^{* * * * *}$ Investigador del Instituto de Investigación para el Desarrollo sustentable de Ceja de Selva INDES-CES, Universidad Nacional Toribio Rodríguez de Mendoza de Amazonas, Perú. ORCID: https://orcid.org/0000-00029670-0970. E-mail: soliva@indes-ces.edu.pe

Recibido: 13/10/2020

Acept ado: 10/12/2020 


\section{Influence of substrates on the growth and development of tomato (Lycopersicum esculentum Mill) grown under a hydroponic system in a greenhouse}

ABSTRACT

The present work aimed to evaluate the influence of substrates in the growth and development of tomato (Lycopersicum esculentum Mill) grown under a hydroponic system in a greenhouse. It was installed under a complete randomized design (DCA), with four treatments: $\mathrm{Tl}$ (75\% rice husk $+25 \%$ sand, T2 (75\% carbonized rice husk $+25 \%$ sand, T3 (75\% Sawdust $+25 \%$ sand, T4 ( $40 \%$ charred rice husk $+35 \%$ sawdust $+25 \%$ sand); 8 repetitions and 32 plants. It was found that T2 favored the increase in height $(139.13 \mathrm{~cm})$, stem diameter $(10.95 \mathrm{~mm})$, number of first quality tomatoes (15.25) and yield (3.03 kg / plant); however, T3 and Tl showed low influence on these variables. It is concluded that the tomato obtains greater morphological and physiological characteristics in substrate (charred husk + sand); sawdust as a substrate is not ideal for its growth and development.

KEYWORDS: charred husk; hydroponics; tomato yield; inert substrate; clean technology

Introducción

El incremento de la población mundial obliga principalmente al sector agrícola a generar nuevas tecnologías con la finalidad de aumentar el rendimiento y calidad alimenticia para el mercado demandante (Saath \& Fachinello, 2018).

El tomate es la hortaliza más cultivada, en diferentes regiones del mundo, sin embargo, requiere mucho de condiciones como clima, suelo, calidad de agua y manejo agronómico adecuadas para su desarrollo (Victoria et al., 2011).

El requerimiento de dichas condiciones, es un factor limitante para el cultivo en muchos lugares; sin embargo, a raíz de este problema, el cultivo hidropónico, surge como una alternativa muy viable para el tomate, ya que este sistema permite crear condiciones ambientales y medios de cultivo (sustratos) favorables para las plantas (Bastida, 2002).

Con la hidroponía, se ahorra agua y nutrientes, logrando mejorar los rendimientos, disminución de costos de producción y reducción de las descargas de fertilizantes al ambiente (Castillo, 2014). Dentro de este sistema, la solución nutritiva es uno de los elementos más 
importantes, una adecuada selección de los minerales resulta ser la base para el éxito del cultivo (López, 2018). En efecto, una fertilización en exceso puede afectar considerablemente el crecimiento de la planta y estado fisiológico del fruto (Romero et al., 2009)

Otro elemento, indispensable es el sustrato, que sirve como anclaje y gracias a sus propiedades físicas, favorece el desarrollo de las plantas (Hartmann et al., 2014). Los sustratos, son diversos y variables, la selección de alguno de ellos, depende de su disponibilidad y del tipo la especie a cultivar (Abad \& Noguera, 2000).

Un sustrato muy utilizado en los sistemas hidropónicos, es la cascarilla de arroz carbonizada, gracias a sus múltiples propiedades que destacan, como la alta durabilidad, contenido de sílice, densidad aparente (0,090 y 0,22 g/ml), pH neutro, conductividad eléctrica y su baja capacidad de intercambio catiónico (CIC), siendo comparado muchas veces con un suelo agrícola, por la similitud con algunas de sus propiedades físicas (Yu et al., 2008; Olle et al., 2012).

Aunque Ortega et al. (2010), menciona que el aserrín de madera, también se puede utilizar como sustrato, por algunas características particulares como aireación, porosidad y retención de agua, favorece la estabilidad de $\mathrm{pH}$ y mayor retención de nutrientes. Aunque, no se reportan muchos antecedentes, Handreck \& Black (2005), mencionan que, en los últimos 25 años, este subproducto de la industria maderera, puede competir con otros sustratos, pero su uso debe ser después de un proceso de deshidratación (Maher et al., 2008).

En la región Amazonas y gran parte de Perú, es común el uso de cascarilla de arroz sin carbonizar como sustrato para un sistema hidropónico, a pesar que muchas veces no son adecuados para ciertos cultivos, por lo que, con el presente trabajo se busca evaluar la influencia de diferentes sustratos en el crecimiento y desarrollo de tomate (Lycopersicum esculentum Mill) cultivado bajo un sistema hidropónico bajo invernadero.

\section{Aspectos geográficos del sitio de estudio}

La investigación se desarrolló en los ambientes de invernadero de la Estación Experimental de la Universidad Nacional Toribio Rodríguez de Mendoza con sede en el distrito Huambo, a través del Instituto de Investigación para el Desarrollo Sustentable de Ceja de Selva, 
con coordenadas latitud $06^{\circ} 25^{\prime} 39^{\prime \prime}$ Sur y longitud $77^{\circ} 32^{\prime} 13^{\prime \prime}$ Oeste y altitud de 1714 m.s.n.m. La temperatura promedio en invernadero fue de $25^{\circ} \mathrm{C}$ y una humedad relativa promedio de $60 \%$.

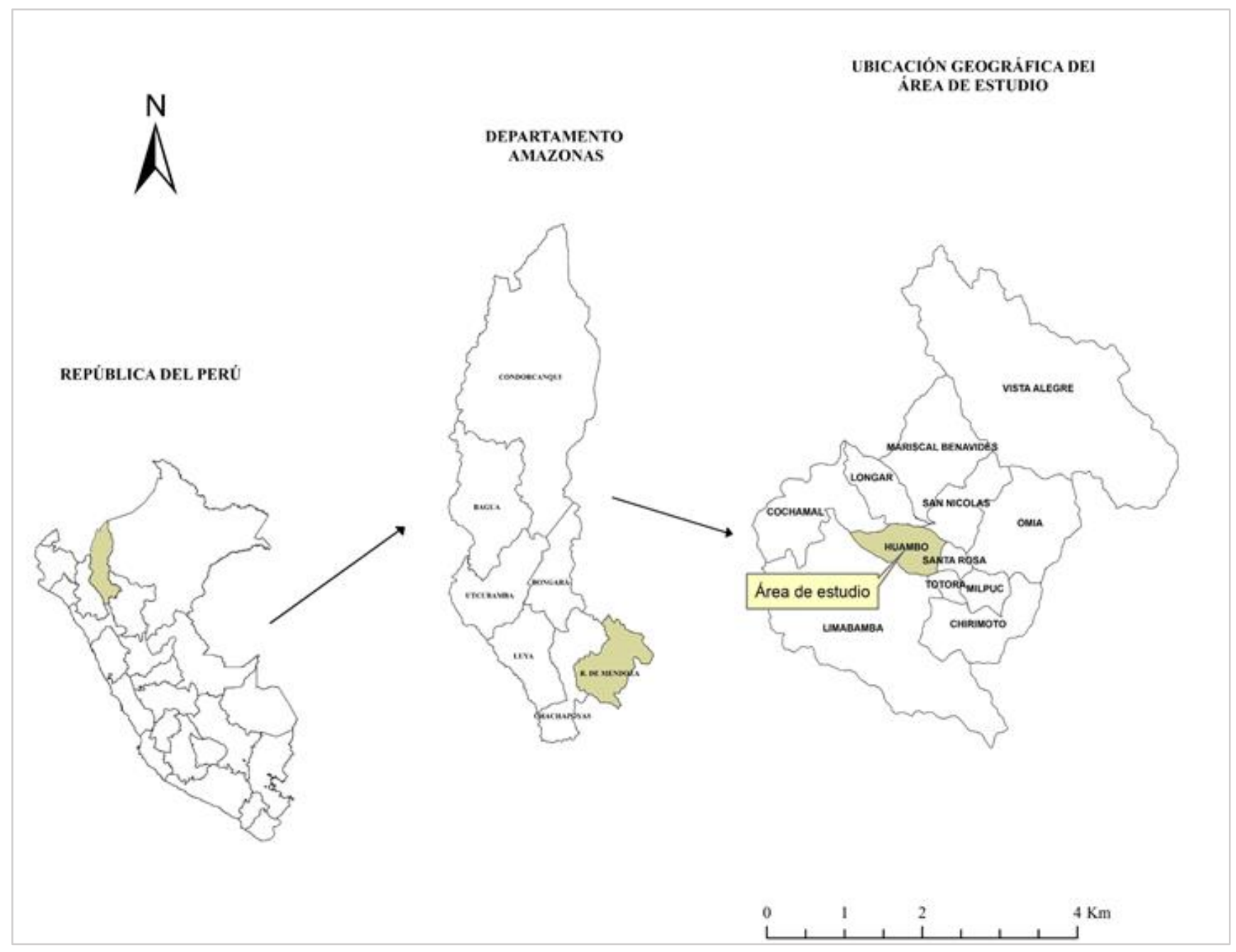

Figura 1. Área de estudio situado en el caserío de Miraflores distrito Huambo (provincia de Rodríguez de Mendoza).

2. Materiales y métodos

2.1. Diseño de la investigación y tratamientos

La investigación se instaló bajo un diseño completo al azar (DCA) con 4 tratamientos, 8 repeticiones y 32 plantas en total. 
REVISTA DE LA UNIVERSIDAD DEL ZULIA. 3ㄹépoca. Año $12 \mathrm{~N}^{\circ}$ 32, 2021

Gelver Silva Valqui et al. // Influencia de sustratos en el crecimiento y desarrollo de tomate ...317-329

DOI: http://dx.doi.org/10.46925//rdluz.32.19

Tabla 01. Descripción de tratamientos utilizados en plantas de L. esculentum Mill, en invernadero

\begin{tabular}{cl}
\hline Tratamientos & Descripción \\
\hline T1 & $75 \%$ cascarilla de arroz $+25 \%$ arena \\
T2 & $75 \%$ cascarilla de arroz carbonizada $+25 \%$ arena \\
T3 & $75 \%$ Aserrín $+25 \%$ arena, \\
T4 & $40 \%$ cascarilla de arroz carbonizada $+35 \%$ aserrín $+25 \%$ arena \\
\hline
\end{tabular}

2.2. Carbonizado de cascarilla de arroz

Se construyó un horno artesanal, para ello se utilizó un cilindro 200 litros galvanizado, se cortó por la mitad en forma transversal; se hizo un agujero sobre la tapa y se colocó un tubo galvanizado de 2 pulgadas, que sirvió de chimenea, así mismo se realizó varios agujeros al rededor del latón para facilitar la oxigenación. En el interior del horno se colocaron trozos de madera y se prendió fuego, hasta que se forme la braza; encima se dispersó la cascarilla de arroz, hasta cubrir el horno por completo. Durante el proceso se realizó un volteado constante para garantizar un quemado homogéneo. Luego de 5 horas, cuando la cascarilla presentó un quemado uniforme, se retiró el fuego, además se aplicó un riego controlado, para evitar un mayor quemado del sustrato (Velázquez-Maldonado et al., 2019).

\subsection{Fermentado y desinfección de cascarilla de arroz}

En un cilindro de 200 litros se depositó la cascarilla de arroz hasta las 3/4 partes del recipiente, luego se llenó hasta el aforo con agua. Se adicionó hipoclorito de sodio al 5 \%, dejándolo en reposo por 24 horas. Transcurrido el tiempo, se lavó la cascarilla con abundante agua, finalmente se colocó sobre un plástico de polietileno para drenar el exceso de humedad retenida.

\subsection{Desinfección de arena}

La arena utilizada fue de cerro, éste se depositó en una carretilla, luego se agregó agua hervida $\left(100{ }^{\circ} \mathrm{C}\right)$. Con una pala se realizó un volteado para que la esterilización sea homogénea. Se dejó en reposo por un tiempo de 10 minutos, repitiendo el procedimiento por tres veces; 
finalmente la arena lavada fue depositada sobre mantas de polietileno para drenar el exceso de agua.

\subsection{Caracterización de propiedades físicas de sustratos}

En laboratorio se realizó las combinaciones de sustratos, según los tratamientos en estudio, éstos se llenaron en bolsas de polietileno de 12.7 x $20.32 \mathrm{~cm}$ de ancho y largo respectivamente, se utilizaron 10 bolsas para cada sustrato. Se colocaron sobre un recipiente de polietileno, para captar el excedente de agua producto del drenaje. Con una probeta se agregó agua a los sustratos hasta que éstos queden en capacidad de campo, determinado los siguientes parámetros:

Porosidad total del sustrato-PS $(\%)=\frac{B}{A} * 100$

Capacidad de aireación del sustrato - AS $(\%)=\frac{A}{C} * 100$

Retención de agua $(\%)=\frac{P T S}{C A S}$

Dónde:

A: Volumen del sustrato; B: Volumen de agua agregada al sustrato; C: Volumen de agua perdido; PTS: Porosidad total del sustrato; CAS: Capacidad de aireación del sustrato.

\subsection{Almacigado de tomate}

La variedad de tomate utilizado fue Abigail; las semillas se colocaron en sustrato comercial Jiffy (fibra de coco + turba) combinado con arena, proporción 2:1 v/v respectivamente. Éste estuvo contenido en bandejas de germinación de polietileno, capacidad de 72 cavidades, colocando 2 semillas por golpe.

2.7. Llenado de sustratos en mangas de polietileno y colocado en invernadero

Las mangas de polietileno fueron cortadas a un tamaño de $90 \mathrm{~cm}$, cerrándola en un extremo con grampas dándole una forma de bolsa. Por consiguiente, se colocaron los sustratos con una distribución uniforme para evitar cámaras de aire. Las mangas llenadas fueron acomodadas en hileras tipo surco mellizo en invernadero; luego con una navaja se procedió a 
realizar cortes circulares en la superficie con distancias de $30 \mathrm{~cm}$ entre sí, para el sembrado de las plántulas.

\subsection{Instalación de sistema de riego}

Se colocó una manguera de riego de $16 \mathrm{~mm}$ en el centro de las hileras formados por las mangas, luego se colocaron goteros auto compensantes con microtubos, para cada hoyo.

\subsection{Trasplante de plántulas de tomate}

Después de realizar un riego a capacidad de campo a los sustratos, se realizó el trasplante de plántulas, colocándolas junto con el sustrato rizosférico procedente del germinador. El tamaño de la plántula sembrada fue $10 \mathrm{~cm}$.

\subsection{Riego y fertilización}

Para el riego se construyó un tanque y sistema de riego por bombeo, donde se mezclaron las soluciones hidropónicas ya formuladas (A y B) según el protocolo de la Universidad Nacional Agraria la Molina. El intervalo de riego fue inter diario, con una frecuencia de 3 minutos.

\subsection{Entutorado y podas de formación de tomate}

Se utilizaron cuerdas de nylon y ganchos comerciales, éstos fueron colocado en la base del tallo de la planta en sus inicios y en las partes axilares durante la fase fenológica del tomate. Fueron fijados en una red de alambre galvanizado sobre postes en "T" de $2 \mathrm{~m}$. Las podas fueron principalmente de ramas axilares y hojas para dar forma a la planta y optimizar su crecimiento.

\subsection{Variables evaluadas}

Altura de planta: se tomaron medidas a inicios con una regla milimetrada de metal y luego con wincha, desde la base de la planta hasta la base del ápice de la misma (Burbano, 2017).

Diámetro de tallo: se tomaron medidas del diámetro de tallo de tomate, según consideraciones a $1 \mathrm{~cm}$ del cuello de la planta, con ayuda de un vernier digital, realizando cada 30 días.

Número de frutos: se registró contabilizando el número de frutos en estado de madurez comercial y de acuerdo a su calibre (primera, segunda y tercera). 
REVISTA DE LA UNIVERSIDAD DEL ZULIA. 3ㄹépoca. Año $12 \mathrm{~N}^{\circ}$ 32, 2021 Gelver Silva Valqui et al. // Influencia de sustratos en el crecimiento y desarrollo de tomate ...317-329

DOI: http://dx.doi.org/10.46925//rdluz.32.19

Peso de frutos: se estimó los pesos de frutos con estado de madurez comercial, con la ayuda de una balanza digital capacidad $5 \mathrm{~kg}$.

\subsection{Análisis estadístico de los datos}

Los datos morfológicos de tomate fueron normales y homogéneos, para ello se realizó el análisis de varianza ANOVA $(\alpha<0.05)$ y la comparación múltiple de medias con el test post-hoc de Tukey al 5\% de nivel de significancia. Se utilizó el software estadístico InfoStat versión 2017.

\section{Resultados}

El análisis de varianza muestra que los sustratos tuvieron efectos significativos para las variables altura, diámetro y rendimiento en plantas de tomate ( $p$-valor < 0.05). No obstante, para la variable número de frutos por planta y tratamiento no mostró efectos significativos (tabla 02).

Tabla 02. Análisis de varianza de acuerdo a los tratamientos

\begin{tabular}{lccccccc}
\hline \multirow{2}{*}{ FV } & & & & & & \multicolumn{2}{c}{ NF (calibre en mm) } \\
\cline { 6 - 8 } & $\mathrm{H}$ & DT $(\mathrm{cm})$ & NTFM & $\begin{array}{c}\text { RDTO } \\
(\mathrm{kg} / \mathrm{planta})\end{array}$ & $\begin{array}{l}\text { Primera } \\
(72-81)\end{array}$ & $\begin{array}{l}\text { Segunda } \\
(62-71)\end{array}$ & $\begin{array}{l}\text { Tercera } \\
(53-61)\end{array}$ \\
\hline Tratamiento & $0.0001^{*}$ & $0.0102^{*}$ & $0.3417 \mathrm{~ns}$ & $0.0057^{*}$ & $0.0098^{*}$ & $0.6020 \mathrm{~ns}$ & $0.8929 \mathrm{~ns}$ \\
\hline
\end{tabular}

*= Diferencia significativa; ns: no significativo; FV: fuente de variación; H: altura; DT: diámetro de tallo; NTFM: número total de frutos maduros; RDTO: rendimiento; NF: número de frutos

La tabla 03, muestra el test de Tukey $(\alpha=0.05)$, en la que T2 (75\% cascarilla de arroz carbonizada $+25 \%$ arena) mostró superioridad en las variables altura de planta, diámetro de tallo, rendimiento de tomate y número de frutos de primera calidad, seguido por el T4 (40\% cascarilla de arroz carbonizada + 35\% aserrín + 25\% arena). No obstante, el T3 (75 \% Aserrín + $25 \%$ arena) no mostró efetos positivos en las variables altura y diámetro, así mismo resultó ser el segundo peor tratamiento en rendimiento junto al $\mathrm{Tl}$ (75\% cascarilla de arroz $+25 \%$ arena) (figura 2). 
REVISTA DE LA UNIVERSIDAD DEL ZULIA. 3época. Año $12 \mathrm{~N}^{\circ}$ 32, 2021 Gelver Silva Valqui et al. // Influencia de sustratos en el crecimiento y desarrollo de tomate ...317-329

DOI: http://dx.doi.org/10.46925//rdluz.32.19

Tabla 03. Comparación múltiple de medias según el test de Tukey $(\alpha=0.05)$ para las características morfológicas y rendimiento de L. esculentum Mill, bajo diferentes sustratos

\begin{tabular}{ccccc}
\hline \multirow{2}{*}{ Tratamientos } & $\mathrm{H}$ & $\mathrm{DT}(\mathrm{cm})$ & RDTO $(\mathrm{kg} /$ planta) & NF (calibre en mm) \\
\cline { 3 - 5 } & & & & Primera (72-81) \\
\hline T1 & $121.25 \mathrm{bc}$ & $9.68 \mathrm{ab}$ & $2.04 \mathrm{~b}$ & $7.75 \mathrm{~b}$ \\
$\mathrm{~T} 2$ & $139.13 \mathrm{a}$ & $10.95 \mathrm{a}$ & $3.03 \mathrm{a}$ & $15.25 \mathrm{a}$ \\
$\mathrm{T} 3$ & $107.00 \mathrm{c}$ & $9.10 \mathrm{~b}$ & $2.20 \mathrm{~b}$ & $9.00 \mathrm{ab}$ \\
T4 & $127.13 \mathrm{ab}$ & $9.71 \mathrm{ab}$ & $2.8 \mathrm{ab}$ & $14.69 \mathrm{ab}$ \\
\hline
\end{tabular}

H: altura; DT: diámetro de tallo; RDTO: rendimiento; NF: número de frutos. Medias con una letra común no son significativamente diferentes ( $p>0.05)$.

En la tabla 04, se observa que todos los sustratos presentaron una porosidad media, sin embargo, el Tl (75 \% cascarilla de arroz + $25 \%$ arena), mostró mayor aireación, seguido por el T3 (75\% Aserrín + 25\% arena); por su parte, el T4 (40\% cascarilla de arroz carbonizada + $35 \%$ aserrín + $25 \%$ arena) presentó menor aireación, por ende, presentó mayor capacidad de retención de humedad. La retención de humedad no tuvo relación directa con la aireación, ya que no siempre una menor porosidad tuvo una mayor retención de humedad.

Tabla 04. Propiedades físicas evaluadas de la combinación de sustratos

\begin{tabular}{cccc}
\hline Tratamientos & Porosidad (\%) & Aireación (\%) & $\begin{array}{c}\text { Retención de humedad } \\
(\%)\end{array}$ \\
\hline T1 & 50 & 27.0 & 23.0 \\
T2 & 50 & 8.0 & 42.0 \\
T3 & 50 & 21.7 & 28.3 \\
T4 & 50 & 6.7 & 43.3 \\
\hline
\end{tabular}

\section{Discusión}

El sustrato cascarilla de arroz carbonizada + arena, incrementó considerablemente la altura de planta, diámetro de tallo, número de frutos de primera calidad y rendimiento, superando largamente a los demás sustratos (aserrín y cascarilla sin carbonizar); así mismo la cascarilla carbonizada combinado con aserrín, también se mostró superior a los otros sustratos. 
Bracho, 2009), asevera que la cascarilla cruda, por lo general favorece la retención de humedad, pero, éste repotencia su capacidad cuando son mezclados con otros sustratos (Schmilewski, 2001), tales como arena (Bunt, 1988).

Águila (2008), menciona que el uso de cascarilla carbonizada como sustrato para sistemas hidropónicos, resulta ser muy beneficioso, gracias a sus características químicas (contenido de Sílice $\left(\mathrm{SiO}_{2}\right.$ ) en las cenizas $80.33 \%$, macro y micro nutrientes) (Velázquez-Maldonado et al., 2019) y por sus características físicas (Quintero et al., 2011). Dicha afirmación es complementada por Peña et al. (2013), donde encontraron que la cascarilla carbonizada superó en $27.3 \%$ a la cascarilla cruda y a cielo abierto, en la variable rendimiento de tomate de primera calidad.

La capacidad de los sustratos para la retención de humedad, aireación, densidad aparente baja, porosidad total, debe ser mojable y buen drenaje $40 \%$, influyen positivamente en el desarrollo de la planta (Cadahía, 2005; AlShrouf, 2017, Rodríguez et al., 2018), siendo corroborada en la presente investigación, donde cascarilla carbonizada fue más influyente gracias a sus características físico-químicas.

Las propiedades físicas de los sustratos, son aspectos importantes en un cultivo hidropónico, ya que un sustrato inadecuado puede ocasionar pérdida de nutrientes por drenaje, hasta en un $50 \%$ del total aplicado (Arredondo et al., 2008), siendo poco aprovechada por la planta. Hao y Papadopoulos (2002), registraron que, dentro de las pérdidas de nutrientes resaltan algunos macros y micro nutrientes esenciales para el óptimo desarrollo de tomate.

Cruz (2009), menciona que la pérdida de nutrientes, afecta significativamente los rendimientos, ya que éstos guardan una relación directa con dichos factores. Por el contrario, De Grazia et al. (2007), afirma que algunos sustratos gracias a su contenido natural de ciertos minerales, pueden mitigar el impacto de pérdidas nutrimentales y en asociatividad con otros nutrientes adicionados éstos pueden incrementar significativamente los rendimientos en los cultivos. Similar a lo observado en la investigación con la cascarilla de arroz carbonizada.

Por su parte, se observó la baja influencia del sustrato aserrín, que, a pesar de tener buena capacidad de retención de humedad, generó un efecto ditrimental para las plantas de tomate, es probable que esto haya ocurrido por una alta acumulación de sales, generando un desbalance nutricional (Papadopoulos, 1991); también es probable que haya ocurrido un efecto alelopático, 
REVISTA DE LA UNIVERSIDAD DEL ZULIA. 3época. Año $12 \mathrm{~N}^{\circ}$ 32, 2021 Gelver Silva Valqui et al. // Influencia de sustratos en el crecimiento y desarrollo de tomate ...317-329

DOI: http://dx.doi.org/10.46925//rdluz.32.19

ya que Maher et al. (2008), mencionan que, el aserrín debe pasar por un proceso de deshidratación antes de ser utilizados como sustratos para hidroponía.

\section{Conclusiones}

El tomate cultivado bajo un sistema hidropónico, muestra buen crecimiento y desarrollo cuando son sembrados en sustrato (cascarilla carbonizada + arena 75:25 v/v respectivamente); sin embargo, el aserrín y cascarilla de arroz sin carbonizar, no son sustratos ideales, para ser utilizados en este cultivo y bajo un sistema tecnificado.

\section{Referencias}

Abad B. M., \& Noguera M. P. (2000). Los sustratos en los cultivos sin suelo, pp: 137-183. Manual de Cultivo sin Suelo. URRESTARAZU G., M.(Ed.). Mundi-Prensa. Madrid, España.

Águila, I., \& Sosa, M. (2008). Evaluación físico químico de cenizas de cascarilla de arroz, bagazo de caña y hoja de maíz y su influencia en mezclas de mortero, como materiales puzolánicos. Revista de la Facultad de Ingeniería Universidad Central de Venezuela 23(4): 55-66.

AlShrouf, A. (2017). Hidroponía, aeroponía y acuaponia en comparación con la agricultura convencional. Revista estadounidense de investigación científica de ingeniería, tecnología y ciencias (ASRJETS) 27 (1): 247-255.

Arredondo, J. L. O., Ramos, J. Z. C., Ramos, J. J. M., González, G. A., Chávez, L. T., Tapia, P. V., $\&$ Reyes, S. E. (2008). Eficiencia de suelo y tezontle en sistemas de producción de tomate en invernadero. Revista Fitotecnia Mexicana 31(4): 367-374.

Bastida, T. (2002). Sustratos hidropónicos. Departamento de Preparatoria Agrícola, Universidad Autónoma Chapingo, Chapingo, México.

Bracho, J., Pierre, F., \& Quiroz, A. (2009). Caracterización de componentes de sustratos locales para la producción de plántulas de hortalizas en el estado Lara, Venezuela. Bioggro 21(2): 117-124.

Bunt, A.C. (1988). Media and mixes for container-grown plants. Unwin Hyman Ltd., Great Britain. 309 p.

Burbano, E. (2017). Producción de líneas de tomate "chonto", Solanum lycopersicum Mill., con expresión del gen sp responsable del crecimiento determinado. Revista Colombiana de Ciencias Hortícolas 1l(1): 63-71.

Cadahía López, C. (2005). Fertirrigación. Cultivos hortícolas, frutales y ornamentales.: cultivos hortícolas, 
frutales y ornamentales. Mundi-prensa Libros.

Castillo, S. D., Moreno-Pérez, E. D. C., Pineda-Pineda, J., Osuna, J. M., Rodríguez-Pérez, J. E., \& Osuna-Encino, T. (2014). Producción hidropónica de jitomate (Solatium lycopersicum L.) con y sin recirculación de la solución nutritiva. Agrociencia 48(2): 185-197.

Cruz Crespo, E., Sandoval Villa, M., Volke Haller, V. H., Can Chulim, Á., \& Sánchez Escudero, J. (2012). Efecto de mezclas de sustratos y concentración de la solución nutritiva en el crecimiento y rendimiento de tomate. Revista mexicana de ciencias agrícolas 3(7): 1361-1373.

De Grazia, J., Tittonell, P. A., \& Chiesa, A. (2007). Efecto de sustratos con compost y fertilización nitrogenada sobre la fotosíntesis, precocidad y rendimiento de pimiento (Capsicum annuurn). Ciencia e investigación agraria 34(3): 195-204.

Handreck, KA, Black, ND y Black, N. (2002). Medios de cultivo para plantas ornamentales y césped. Prensa UNSW.

Hao X, A P Papadopoulos (2002) Growth, photosynthesis and productivity of greenhouse tomato cultivated in open o closed rockwool systems. Can. J. Plant Sci. 82:771-780.

Hartmann HT, DE Kester, FT Davies, RL Geneve. (2014). Hartmann \& Kester's Plant Propagation:Principles and Practices. Eigth Edition. United States of America. Pearson. 922 p.

López Elías, J. (2018). La producción hidropónica de cultivos. Idesia (Arica) 36(2): 139-141.

Maher, M., Prasad, M., \& Raviv, M. (2008). Organic soilless media components. Soilless culture: Theory and practice 459-504.

Olle, M., Ngouajio, M., \& Siomos, A. (2012). Vegetable quality and productivity as influenced by growing medium: A review. Zemdirbyste 99(4): 399-408.

Ortega Martinez, L. Sanchez Olarte, J. Diaz Ruiz, R. Ocampo Mendoza, J. (2010). TOMATE ( Lycopersicum esculentum MILL ) proporción al aumento de la superficie cultivada . producción en la aportación de divisas a la. Ra Ximhai 6: 365-372. https://doi.org/10.1016/j.actaastro.2008.03.010

Papadopoulos, A. P. (1991). Growing greenhouse tomatoes in soil and in soilless media. Available from Communications Branch, Agriculture Canada.

Peña, M. Y., Posada, F. C., \& Monsalve, O. I. (2013). Producción hidropónica de tomate (Solanum lycopersicum L.) en cascarilla de arroz mezclada con materiales minerales y orgánicos. Revista Colombiana de Ciencias Hortícolas 7(2): 217-227.

Quintero, M. F., González, C. A., \& Guzmán, J. M. (2011). Sustratos para cultivos hortícolas y flores de corte. Flórez R., VJ (ed). Sustratos, Manejo del Clima, Automatización y Control en Sistemas de 
Cultivo sin Suelo. Universidad Nacional de Bogotá. Colombia.pp, 79-108.

Rodríguez, R. D. L., Lara-Herrera, A., Padilla-Bernal, L. E., Avelar-Mejía, J. J., \& España-Luna, M. P. (2018). Proporción de drenaje de la solución nutritiva en el rendimiento y calidad de tomate en hidroponía. Revista mexicana de ciencias agrícolas 9(20): 4343-4353.

Romero, M. V., Terraza, S. P., Peña, P. S., Verdugo, S. H., Enciso, T. O., Madrid, J. L. C., \& Bojorquez, A. D. A. (2009). Fertirrigación con diferentes formas de nitrógeno en el cultivo de tomate en un suelo arcilloso. Interciencia 34(2): 135-139. ISSN: 0378-1844

Saath, K. C. D. O., \& Fachinello, A. L. (2018). Crescimento da demanda mundial de alimentos e restrições do fator terra no Brasil. Revista de Economia e Sociologia Rural 56(2): 195-212.

Schmilewski, G. (2001). Limitations of additional organic material in growing media. FlowerTech 4(2): 20-23.

Velázquez-Maldonado, J., Juárez-López, P., Anzaldo-Hernández, J., Alejo-Santiago, G., ValdezAguilar, L. A., Alia-Tejacal, I., ... \& Guillén-Sánchez, D. (2019). Concentración nutrimental de biocarbón de cascarilla de arroz. Revista fitotecnia mexicana 42(2): 129-136.

Victoria, NG, van der Valk, OMC y Elings, A. (2011). Horticultura Protegida Mexicana:Produccióny mercado de horticultura protegida mexicana descrita y analizada (No. 1126). Wageningen UR Horticultura en invernadero / LEI.

Yu, C., Liu, H., Xing, Y., Manukovsky, N. S., Kovalev, V. S., \& Gurevich, Y. L. (2008). Bioconversion of rice straw into a soil-like substrate. Acta Astronautica 63(7-10): 1037-1042. https://doi.org/10.1016/j.actaastro.2008.03.010 